\title{
Ligustilide modulates oxidative stress, apoptosis, and immunity to avoid pathological damages in bleomycin induced pulmonary fibrosis rats via inactivating TLR4/MyD88/NF-KB P65
}

\author{
Shu Luo ${ }^{\#}$, Junzuo Gong ${ }^{\#}$, Xiaoping Cao, Shiping Liu \\ Department of Emergency, Affiliated Hospital of North Sichuan Medical College, Nanchong, China \\ Contributions: (I) Conception and design: S Liu; (II) Administrative support: S Liu; (III) Provision of study materials or patients: X Cao; (IV) \\ Collection and assembly of data: All authors; (V) Data analysis and interpretation: S Luo, J Gong; (VI) Manuscript writing: All authors; (VII) Final \\ approval of manuscript: All authors. \\ \#These authors contributed equally to this work. \\ Correspondence to: Shiping Liu. No. 63 Cultural Road, Shunqing District, Nanchong, China. Email: 9532868@qq.com.
}

\begin{abstract}
Background: Pulmonary fibrosis (PF) is a fatal disease with increasing incidence. Ligustilide (LIG) has been shown to inhibit oxidative stress, apoptosis, and inflammation. Here we investigated the possible effect of LIG on bleomycin-induced PF in Sprague-Dawley rats.

Methods: PF rats were set up through a single endotracheal injection of bleomycin ( $5 \mathrm{mg} / \mathrm{kg})$. Then rats were treated with 20, 40, and $80 \mathrm{mg} / \mathrm{kg}$ LIG for four weeks, and the effects were estimated.

Results: Overall, LIG significantly improved ventilation and reduced hyperplasia, and treatment of LIG reduced fibrosis as indicated by Masson staining and reduced expression of transforming growth factorbeta (TGF- $\beta$ ), Fibronectin, and alpha-smooth muscle actin ( $\alpha$-SMA). Oxidative stress was induced with bleomycin while inhibited with LIG, as showed with rebalanced serum lactate dehydrogenase (LDH), and tissue superoxide dismutase (SOD), glutathione peroxidase (GSH) and malondialdehyde (MDA). Apoptosis was further inhibited with LIG, as shown with Terminal dUTP nick-end labeling (TUNEL) staining and expression of Caspase-3, Caspase-9, Bax, and Bcl-2. Th1/Th2 balance was also rebuilt as evaluated with CD4 and IFN $\gamma / \mathrm{IL}-4$ labeled flow cytometry of peripheral blood mononuclear cells (PBMCs) and expression of inducible nitric oxide synthase (iNOS) and IL-10 in the serum and lung. Protein expression of Toll-like receptor 4 (TLR4), HSP60-TLR4-myeloid differentiation factor 88 (Myd88) and nuclear factor-kappa B $(\mathrm{NF}-\kappa \mathrm{B}) \mathrm{p}-\mathrm{P} 65 / \mathrm{P} 65$ was significantly reduced with LIG treatment. All the effects of LIG exhibited in a dosedependent way.

Conclusions: LIG improved bleomycin-induced PF with improved ventilation, reduced fibroblast,

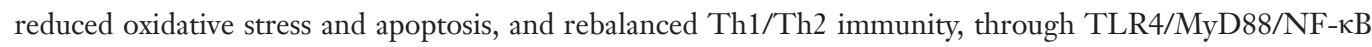
P65 signaling.
\end{abstract}

Keywords: bleomycin; idiopathic pulmonary fibrosis (IPF); ligustilide (LIG); rat

Submitted May 01, 2020. Accepted for publication Jul 04, 2020.

doi: $10.21037 /$ atm-20-4233

View this article at: http://dx.doi.org/10.21037/atm-20-4233

\section{Introduction}

Pulmonary fibrosis (PF), mainly idiopathic pulmonary fibrosis (IPF) is the most common interstitial lung diseases and is a progressive, irreversible, and usually fatal disease (1). With aging and environmental pollution appearing, the incidence of PF increased. However, as a result of limited early diagnosis and intervention, the average life expectancy of IPF patients is $3-5$ years after diagnosis (2). As multiple driven forces promoted $\mathrm{PF}$ with different characters, $\mathrm{PF}$ or IPF are not correct concepts, and pathogenesis of PF 
possibly could be far more than fibrosis. Generally, in PF, aberrantly activated secretion of lung epithelial cells leads to fibroblast proliferation and differentiation into highly active myofibroblasts, which deposit excessive amounts of extracellular matrix (ECM) and irreversibly destroy the lung architecture (3).

Oxidative stress and apoptosis are potential therapeutic targets of PF. Simply elicitation of oxidative stress is sufficient to induce PF in Atp8b1 mutant mice (4). Bleomycin (bleomycin) induced lung injury is the most commonly used model of PF (5). Oxidative stress promoted apoptosis was the target of several potential therapeutics of PF (6). Reduction of oxidative stress, for example, with Sulforaphane significantly reduced PF in C57/BL6 mice (7), while enhancing of oxidative stress promoted toxicity of bleomycin (8). However, the exact influence of oxidative stress and apoptosis in PF patients still needs to be characterized.

Immune responses are essential players in the progression of PF. Dysregulated release of fibroblast cells in PF lung induces a series of pro-inflammatory immune responses, which in turn promotes the progression of $\mathrm{PF}(9)$. The overall inflammatory response in PF closely resembles a Th2 immune response, while lung fibroblasts tend to induce a Th1 immune response (9). Th1/Th2 balance could have essential affects in the regulation of inflammation and apoptosis, thus determining cell fate and progression of $\mathrm{PF}$. Currently, the balance of Th1/Th2 was considered to be regulated with $\mathrm{NF}-\kappa \mathrm{B}, \mathrm{Nrf-2}$, and MyD88 $(7,10,11)$.

Ligustilide (LIG) is the main lipophilic component of the Umbelliferae family in Traditional Chinese Medicine, including Radix angelicae sinensis and Ligusticum chuanxiong (12). LIG has been directly and indirectly shown to inhibit fibrosis in multiple ways. The antioxidant and anti-apoptotic properties of LIG were characterized as neuroprotective (13). In lung tissues, LIG promotes apoptosis of cancer-associated fibroblasts via TLR4 (14) and inhibits pro-inflammatory damage-associated molecular patterns (15). In fibrosis of other organs, LIG inhibition of fibrosis, apoptosis, and inflammation was reported to be related to TLR4, NF-кB, and MyD88 $(16,17)$. SpragueDawley (SD) rats were used in the present study show the possible role of LIG in lung fibrosis by referring to previous researches (18). This research highlighted the possible protective role of LIG in PF. In this study, we aimed to investigate the possible role of LIG in bleomycin-induced fibrosis of rats.

We present the following article in accordance with the
ARRIVE reporting checklist (available at http://dx.doi. org/10.21037/atm-20-4233).

\section{Methods}

\section{Animals and treatments}

All animal experiments complied with the guidelines developed by the Health Department of Local Government and Experimentation Ethics Committee of the Universities and approved by the Department of Health of the Hong Kong Government and the Animal Ethics Committee of The Chinese University of Hong Kong (Ref: 13/085/GRF-5). A total of 30 SD rats (adult, male, 180-220 g) were bought from Shanghai SIPPR-Bk Laboratory Animal Co. Ltd. The rats were raised at $25{ }^{\circ} \mathrm{C}$ with 12 -hour light/dark cycles with relative humidity between $40-70 \%$, fed with standard pellet chow, and had free access to food and water. After acclimatized for a week, rats were randomly assigned to control, bleomycin (marked as bleomycin), bleomycin plus $20 \mathrm{mg} / \mathrm{kg}$ of LIG (marked as LIG $20 \mathrm{mg} / \mathrm{kg}$ ), Bleomycin plus $40 \mathrm{mg} / \mathrm{kg}$ of LIG (marked as LIG $40 \mathrm{mg} / \mathrm{kg}$ ), and Bleomycin plus $80 \mathrm{mg} / \mathrm{kg}$ of LIG (marked as LIG $80 \mathrm{mg} / \mathrm{kg}$ ), groups ( $\mathrm{n}=6$ per group, 2 rats per cage). PF was established through a single endotracheal injection of bleomycin $(5 \mathrm{mg} / \mathrm{kg}$; Zhejiang Hisun Pharmaceutical Co., Ltd., China) in all groups except the control $(5,18)$. After 24 hours, control and bleomycin groups were treated with $2 \mathrm{~mL} / \mathrm{kg} /$ day of saline and other groups with 20, 40, and $80 \mathrm{mg} / \mathrm{kg}$ /day LIG (Sichuan Weikeqi Biotechnology Co., Ltd., China) treatments through intraperitoneal injection for four weeks. Then Lung functions were measured, and rats were sacrificed, and tissues, serum, and blood were collected.

\section{Lung function measurements and sample collection}

After treated for four weeks, Lung function measurements were carried out as described (19). Briefly, a whole-body flow plethysmograph (Buxco Electronics Inc., Wilmington, NC, USA) was used to measure minute ventilation volume directly, and then airway resistance was measured with a FinePointe-series all-in-one Resistance/Compliance (RC) system (Buxco Electronics). After calibration of the equipment with airflow, each SD rat was placed in a small plexiglass container within the plethysmograph chamber and was measured over 5 minutes at room temperature. Then rats were anesthetized with ketamine/xylazine, 
intubated with a sterile cannula that was held in place with surgical thread and then connected to the ventilator with a fixed breathing rate of $145 \mathrm{bpm}$. Airway resistance of each rat was recorded over 3 minutes using Biosystem XA. After measurements, blood was withdrawn by a retroorbital puncture. Then peripheral blood mononuclear cells (PBMCs) were collected from peritoneum with PBS and cultured in DMEM (HyClone, USA) (supplemented with $10 \%$ FBS (PAN, Germany) and 1\% penicillin/streptomycin) at $37{ }^{\circ} \mathrm{C}$ and $5 \% \mathrm{CO}_{2}$. Finally, the anesthetized rat was euthanized following an IACUC approved protocol after the experiment. The blood was centrifuged at $500 \mathrm{~g}$ for 15 minutes, and serums were collected. Left lungs (about $200 \mathrm{mg}$ ) in each group were collected for making tissue homogenate, and right lungs in each group were fixed for making paraffin sections. Samples were temporarily stored at $-80{ }^{\circ} \mathrm{C}$ before the test.

\section{Histology evaluation}

The diced lung tissues were marked, then fixed in 4\% Paraformaldehyde Fix Solution (Beyotime Biotechnology Co., Ltd., China) at $4{ }^{\circ} \mathrm{C}$ overnight. Tissues were then dehydrated in a series of ethanol and xylene solutions and embedded in paraffin. Sections of 4- $\mu$ m thickness were cut then deparaffinized. Hematoxylin and eosin (H\&E Staining Kit, Beyotime Biotechnology Co., Ltd., China) staining and Masson staining were performed according to protocol. Then the slides were observed in $200 \times$ and $400 \times$ scope.

\section{Test of serum lactate debydrogenase (LDH) and tissue superoxide dismutase (SOD), glutathione peroxidase (GSH) and malondialdehyde (MDA)}

The determinations of LDH, SOD, GSH, and MDA in serum or tissues were conducted to evaluate the changes in the oxidant-antioxidant systems. The levels of serum LDH were measured using collected serum and related assay kits (Jiancheng Institute of Biotechnology, Nanjing, China). Lung tissues were sonicated in a $50 \mathrm{mM}$ phosphate buffer, and the supernatant was gained after centrifuged at $3,000 \mathrm{~g}$ for 10 minutes. The supernatant was used to test SOD (tested with SOD assay kit, Jiancheng Institute of Biotechnology, Nanjing, China), GSH (tested with GSHPX assay kit, Jiancheng Institute of Biotechnology, Nanjing, China) and MDA (tested with MDA assay kit, Jiancheng Institute of Biotechnology, Nanjing, China) according to instructions.

\section{Terminal dUTP nick-end labeling (TUNEL)}

The paraffin section of lung tissue was taken to receive conventional dewaxing, antigen repairing, and blocking. Sections were incubated in the working solution $(20 \mu \mathrm{g} / \mathrm{mL}$ proteinase $\mathrm{K}$ dissolving in $10 \mathrm{mmol} / \mathrm{L}$ Tris- $\mathrm{HCl}, \mathrm{PH}$ : 7.4-8; Sigma-Aldrich) at $37^{\circ} \mathrm{C}$ for 15 minutes. Then, $50 \mu \mathrm{L}$ TUNEL reaction solutions (Roche Applied Science, Indianapolis, Inc., IN, USA) were added for incubation at $37{ }^{\circ} \mathrm{C}$ for 1 hour. Next, the sections were washed with PBST, incubated with $100 \mu \mathrm{L}$ diaminobenzidine (DAB) for 30 minutes in converter-peroxidase (POD) $\left(50 \mu \mathrm{L}\right.$, at $\left.37^{\circ} \mathrm{C}\right)$ and wash with PBST. Finally, the slides were observed in 200x scope, four fields of each tissue were randomly harvested, and the average apoptotic cells per 100 cells were analyzed with Image J.

\section{Western blotting}

Tissues were then homogenized in RIPA lysis buffer (Beyotime Biotechnology Co., Ltd., China), tested with a BCA kit (Beyotime Biotechnology Co., Ltd., China) and balanced with $1 \mathrm{X}$ PBS. Then about $30 \mu \mathrm{g}$ of protein for each sample was added with a loading buffer, separated on an SDS-PAGE gel, transferred to a PVDF membrane, and incubated with primary and secondary antibodies. The antibodies used were: anti-Bax (182733), anti-Bcl-2 (196495), anti-Caspase-3 (179517), anti-Caspase-9 (ab184786), antiMyD88 (ab2064), anti-P65 (ab32536) and anti-p-P65 (ab86299) purchased from Abcam; anti-TGF-beta (\#3709), anti-Fibronection (\#4705), anti-alpha-SMA (\#68463), antiTLR4 (\#14358), anti-beta-Actin (\#12748) and all secondary antibodies bought from Cell Signaling Technology.

\section{Flow cytometry}

Flow cytometry was applied to determine $\mathrm{CD} 4^{+} \mathrm{IFN}-\gamma^{+}$and $\mathrm{CD} 4^{+} \mathrm{IL}-4^{+}$lymphocytes balance. The collected PBMCs were cultured in a $5 \% \mathrm{CO}_{2}$ incubator at $37{ }^{\circ} \mathrm{C}$ for 24 hours so that unattached cells were discarded. Then cells were collected in an EP tube. FITC-anti-CD4 was incubated at room temperature in the dark for 30 minutes. Then the cells were washed with cell staining buffer, mixed with $500 \mu \mathrm{L}$ fixation/permeabilization solution (BD, 555028), and incubated in the dark for 45 minutes. Next, 1X BD perm/ wash buffer washed cells were incubated with PE-anti-IL-4 and APC-anti-IFN- $\gamma$ in the dark for 45 minutes, and washed again with $1 \mathrm{X}$ BD perm/wash buffer, and resuspended 
Page 4 of 11
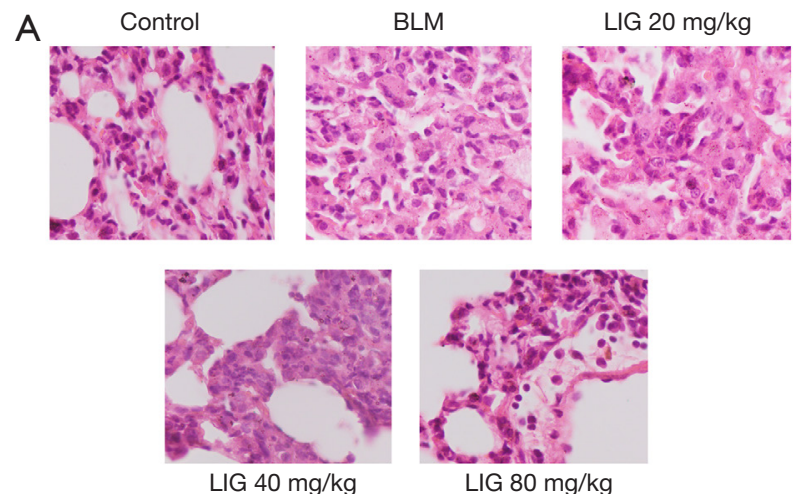

B

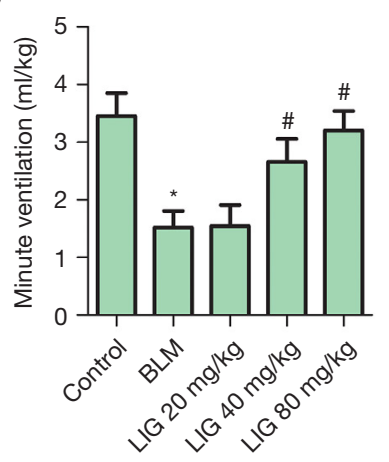

C

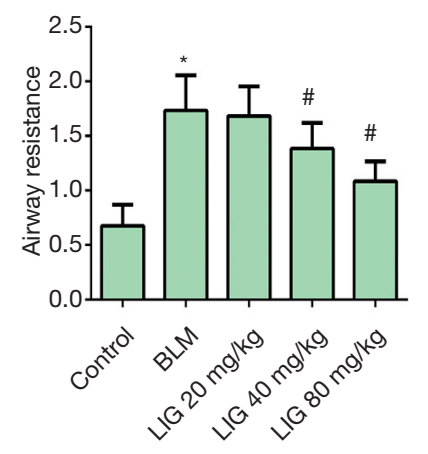

Figure 1 The overall effects of LIG on bleomycin stimulated rat lung. SD rats were treated with saline (Control group) or $5 \mathrm{mg} / \mathrm{kg}$ bleomycin (all treatment group rats) through a single endotracheal injection. Then rats were administered with the indicated dose of LIG or saline for 28 days through intraperitoneal injection. (A) The HE stains of lung tissues were observed under 400x scope. (B) Minute ventilation measured with a whole-body flow plethysmograph. (C) Airway resistance determined with a FinePointe-series all-in-one RC system. $\mathrm{n}=6$ for each group, ${ }^{*} \mathrm{P}<0.05$ versus control and ${ }^{*} \mathrm{P}<0.05$ versus bleomycin with $t$-test. Data were shown as mean \pm SEM. LIG, ligustilide.

with $300 \mu \mathrm{L}$ flow washing liquid. Th1 cells (defined as $\mathrm{CD} 4^{+} \mathrm{IFN}-\gamma^{+}$) and Th2 cells (defined as $\mathrm{CD} 4^{+} \mathrm{IL}-4^{+}$) were detected by flow cytometry (FACSAria; BD Biosciences), and data were analyzed using CellQuest software.

\section{Enzyme-linked immunosorbent assay (ELISA)}

Tissue samples were prepared and balanced as mentioned in western blotting, serum samples were from centrifuged blood. Elisa kits (Cloud-Clone Corp., Wuhan, China) were used to measure the changes in iNOS and IL-10 in rat serum and lung tissue in each group following the instruction. Standard preparation $(50 \mu \mathrm{L})$ was added in the first and second wells, followed by multiple proportion dilution. Sample diluent $(40 \mu \mathrm{L})$ and sample $(10 \mu \mathrm{L})$ were added in each well at $37^{\circ} \mathrm{C}$ for 30 minutes. Washing liquid was then used to wash the wells for five times, and a secondary antibody $(50 \mu \mathrm{L})$ was added in each well at $37^{\circ} \mathrm{C}$ for $30 \mathrm{~min}$. After washed out again, chromogenic agents A and $\mathrm{B}\left(50 \mu \mathrm{L}\right.$ for each) were added in each well at $37^{\circ} \mathrm{C}$ in the dark for 15 minutes. A termination solution $(50 \mu \mathrm{L})$ was added in each well. A microplate reader (Bio-Rad, USA) was used for detection. Sample concentrations were calculated according to the curve of the standard.

\section{Statistical analysis}

All the data were analyzed by Image J and Graphpad Prism 7.0 and presented as the mean \pm standard error of the mean (SEM). Differences were determined with $\mathrm{P}<0.05$ of the $t$-test for at least three independent experiments.

\section{Results}

\section{LIG improved lung function of bleomycin treated rats}

Bleomycin treated rats were administered with 20, 40, and $80 \mathrm{mg} / \mathrm{kg}$ of LIG, respectively, to estimate the overall effects of LIG on PF. HE stains of lung tissues indicated bleomycininduced rats developed lung injury compared to normal individuals, while LIG dose-dependently reduced hyperplasia (Figure 1A). Similar results were seen in minute pulmonary ventilation (Figure 1B) and airway resistance (Figure 1C), as bleomycin significantly reduced minute ventilation and airway resistance, and this was dose-dependently inhibited with LIG $\left({ }^{*} \mathrm{P}<0.05\right.$ versus control and ${ }^{\#} \mathrm{P}<0.05$ versus bleomycin). These revealed that the inhibited lung function with bleomycin could be improved with LIG.

\section{$L I G$ reduced fibrosis of bleomycin treated rats}

$\mathrm{PF}$ of the rats was showed with Masson staining and related expression of TGF- $\beta$, Fibronectin, and $\alpha$-SMA. As expected, bleomycin-induced fibrosis, obviously, and treatment of LIG alleviated the burden of fibrosis (Figure 2A). Similarly, TGF- $\beta$, Fibronectin, and $\alpha$-SMA all were induced with bleomycin while dose-dependently reduced with LIG (Figure $2 B,{ }^{*} \mathrm{P}<0.05$ versus control and ${ }^{\#} \mathrm{P}<0.05$ versus bleomycin). These results revealed that LIG 
A

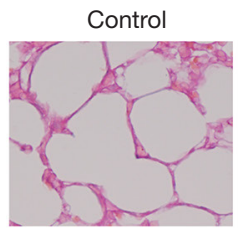

BLM

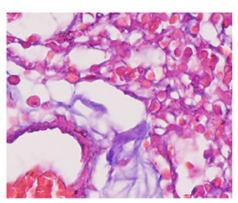

LIG 20 mg/kg

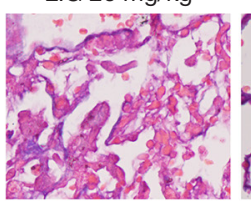

LIG 40 mg/kg

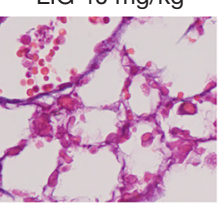

LIG $80 \mathrm{mg} / \mathrm{kg}$

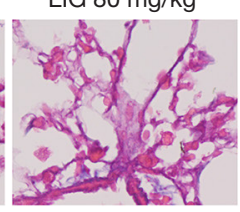

B

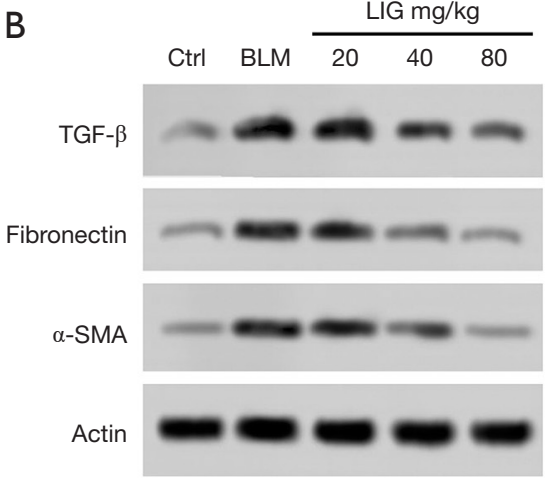

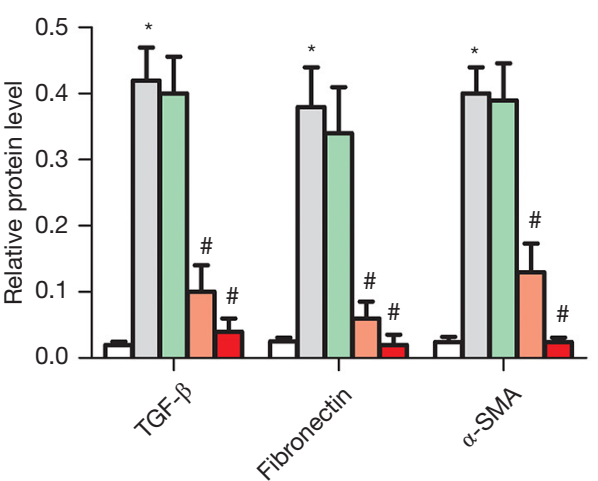

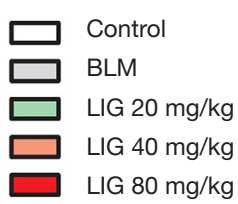

LIG $80 \mathrm{mg} / \mathrm{kg}$

Figure 2 Reduced PF with LIG in bleomycin treated rat. Rats were pretreated with bleomycin once and treated with an indicated dosage of LIG for 28 days. (A) Masson staining of rat lung tissues was observed under $200 \times$ scope. (B) Expression of TGF- $\beta$, Fibronectin, and $\alpha$-SMA was treated with western blotting, and relative protein expression was evaluated with Image $\mathrm{J}$ software. $\mathrm{n}=6$ for each group, ${ }^{*} \mathrm{P}<0.05$ versus control and ${ }^{\#} \mathrm{P}<0.05$ versus bleomycin with $t$-test. Data were shown as mean \pm SEM. PF, pulmonary fibrosis; LIG, ligustilide; TGF- $\beta$, transforming growth factor-beta; $\alpha$-SMA, alpha-smooth muscle actin.

could reduce bleomycin-induced fibrosis of SD rats.

\section{LIG reduced the oxidative stress of bleomycin treated rats}

The relative content of LDH (Figure $3 A$ ) in the serum and SOD (Figure 3B), MDA (Figure 3C) and GSH (Figure 3D) in the tissue were then determined to show the involvement of oxidative stress in LIG improved lung function. Serum LDH and lung MDA were significantly induced with bleomycin, which was dose-dependently reduced with LIG $\left({ }^{*} \mathrm{P}<0.05\right.$ versus control and ${ }^{\#} \mathrm{P}<0.05$ versus bleomycin). The activity of SOD and GSH were significantly inhibited with bleomycin and dose-dependently recovered with LIG ( ${ }^{*} \mathrm{P}<0.05$ versus control and ${ }^{\#} \mathrm{P}<0.05$ versus bleomycin). The reduced $\mathrm{LDH}$ and MDA, together with recovered SOD and GSH, showed that LIG dose-dependently reduced lung oxidative stress-induced with bleomycin in rats.

\section{LIG reduced apoptosis of bleomycin treated rats}

Apoptosis in rat lung was then evaluated with TUNEL staining and expression of apoptosis-related proteins. As indicated in Figure $4 A$, a single dose of bleomycin markedly induced apoptosis of the rat lung in day 28, and treatment LIG significantly reduced apoptotic cells $\left({ }^{*} \mathrm{P}<0.05\right.$ versus control and ${ }^{\#} \mathrm{P}<0.05$ versus bleomycin). The bleomycininduced and LIG reduced apoptosis was further confirmed with western blotting. The ratio of $\mathrm{Bax} / \mathrm{Bcl}-2$, cleaved Caspase-3/Caspase-3 and cleaved Caspase-9/Caspase-9 were all elevated with bleomycin while dose-dependently reduced with LIG (Figure $4 B$, ${ }^{*} \mathrm{P}<0.05$ versus control and ${ }^{\#} \mathrm{P}<0.05$ versus bleomycin).

\section{LIG rebuilt Th1/Th2 balance of bleomycin treated rats}

Th1/Th2 Balance was further evaluated with CD4 and IFN $\gamma /$ IL-4 labeled flow cytometry of PBMCs. CD4 IFN $\gamma^{+}$ cells were markedly induced with bleomycin and dosedependently reduced with LIG (Figure $5 A, B,{ }^{*} \mathrm{P}<0.05$ versus control and ${ }^{\#} \mathrm{P}<0.05$ versus bleomycin). $\mathrm{CD} 4^{+} \mathrm{IL} 4^{+}$cells were not significantly changed with bleomycin, while 40 and 80 $\mathrm{mg} / \mathrm{kg}$ of LIG promoted the amount of $\mathrm{CD}^{+} \mathrm{IL}^{+}$cells (Figure $5 A, C,{ }^{*} \mathrm{P}<0.05$ versus bleomycin). The bleomycin increased, and LIG inhibited Th1 immunity, and the LIG increased Th2 immunity, which was further validated with an expression of iNOS and IL-1 in the blood (Figure 5D) 

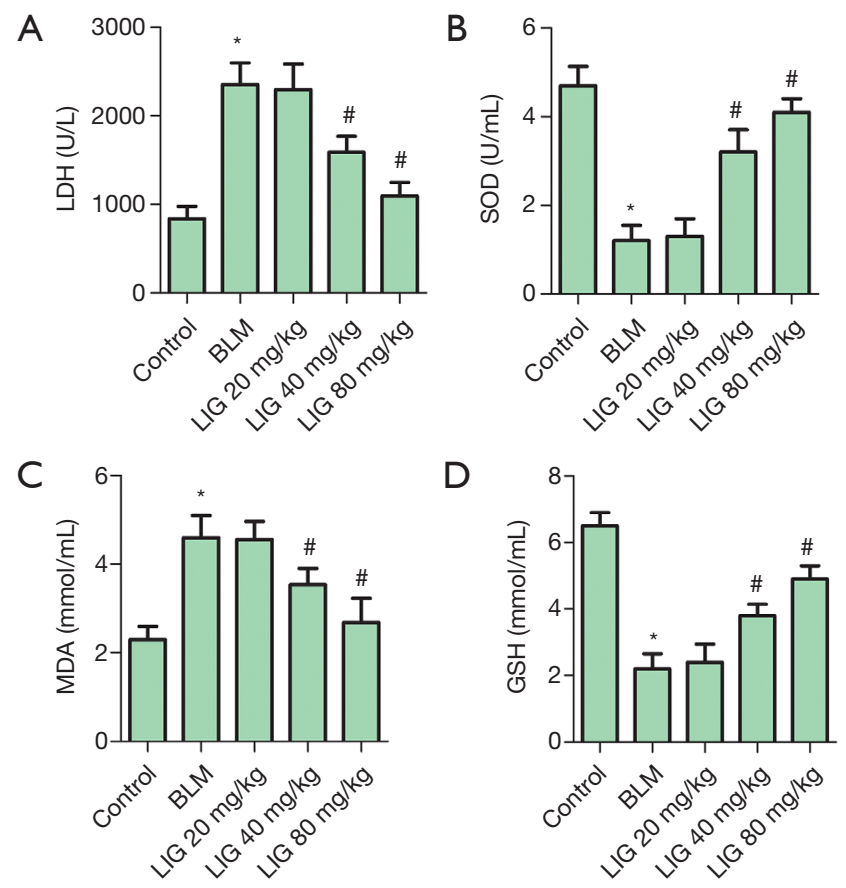

Figure 3 Reduced oxidative stress with LIG in bleomycin treated rat lung. Rats were pretreated with bleomycin once and treated with an indicated dosage of LIG for 28 days and (A) LDH activity in serum, (B) SOD activity in the lung, (C) MDA activity in the lung, and (D) GSH activity in the lung were tested with related kits. $\mathrm{n}=6$ for each group, ${ }^{*} \mathrm{P}<0.05$ versus control and ${ }^{\#} \mathrm{P}<0.05$ versus bleomycin with $t$-test. Data were shown as mean \pm SEM. LIG, ligustilide; LDH, lactate dehydrogenase; SOD, superoxide dismutase; MDA, malondialdehyde; GSH, glutathione peroxidase.

and lung tissue (Figure 5E). Bleomycin obviously induced iNOS both in the serum and lung tissue, and LIG dosedependently reduced iNOS; bleomycin had no significant effects on IL-10, and LIG dose-dependently reduced IL10 both in the serum and lung tissue (Figure $5 D, E,{ }^{*} \mathrm{P}<0.05$ versus control and ${ }^{\#} \mathrm{P}<0.05$ versus bleomycin). These showed the rebalancing of Th1/Th2 with LIG.

\section{LIG reduced fibrosis of bleomycin treated rats through TLR4/MyD88/P65}

The involvement of TLR4 (Figure 6A,B), MyD88 (Figure $6 A, C$ ), and NF-кB P65 (Figure 6A,D) in LIG reduced fibrosis of bleomycin was then investigated with western blotting. The relative expression of TLR-4, MyD88, and

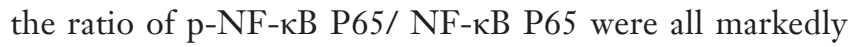
induced with bleomycin, and dose-dependently decreased with LIG $\left({ }^{*} \mathrm{P}<0.05\right.$ versus control and ${ }^{\#} \mathrm{P}<0.05$ versus bleomycin). Results above show that TLR4/MyD88/P65 was involved in LIG reduced fibrosis of bleomycin in SD rats.

\section{Discussion}

Currently, PF remains an irreversible and deadly disease, in which acute exacerbations remain a significant cause of morbidity and mortality (2). Inhibition of fibrosis and maintenance of ventilation was the main issue in treating PF, including IPF. In this study, we showed that LIG dose-dependently reduced the progression of fibrosis and improved ventilation. The results supported LIG as a potential prodrug for $\mathrm{PF}$.

Oxidative stress, pro-inflammatory cytokines, and apoptosis were accumulated, and under PF, inhibition of oxidative stress effectively reduced bleomycin toxicity and PF $(7,20)$. Accumulated oxidative stress would induce mitochondrial dysfunction and apoptosis, which further promoted PF (6). Activation of inflammatory responses is also a key process in PF; for example, the NLRP3 inflammasome is reported to be activated to the promoted progression of bleomycin-induced PF (21), and targeting of NLRP3 inflammasome effectively reduced PF (22). The activation of oxidative stress, inflammation, and apoptosis 
A

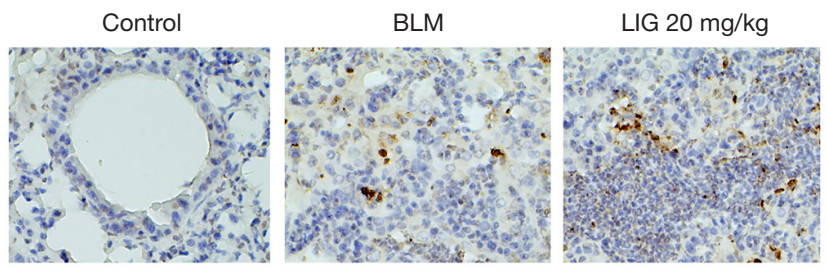

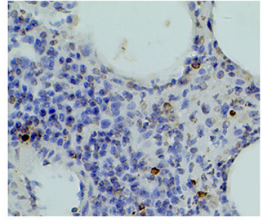

LIG $40 \mathrm{mg} / \mathrm{kg}$

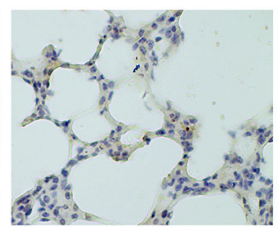

LIG $80 \mathrm{mg} / \mathrm{kg}$

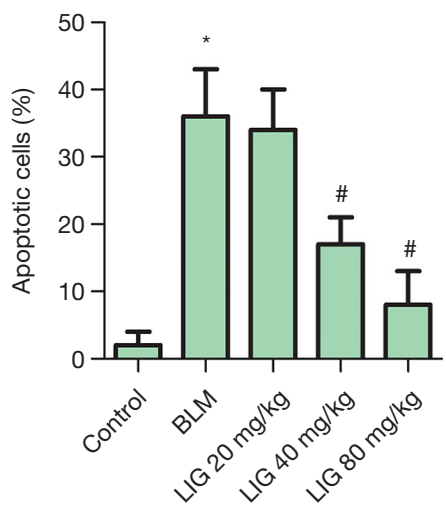

B
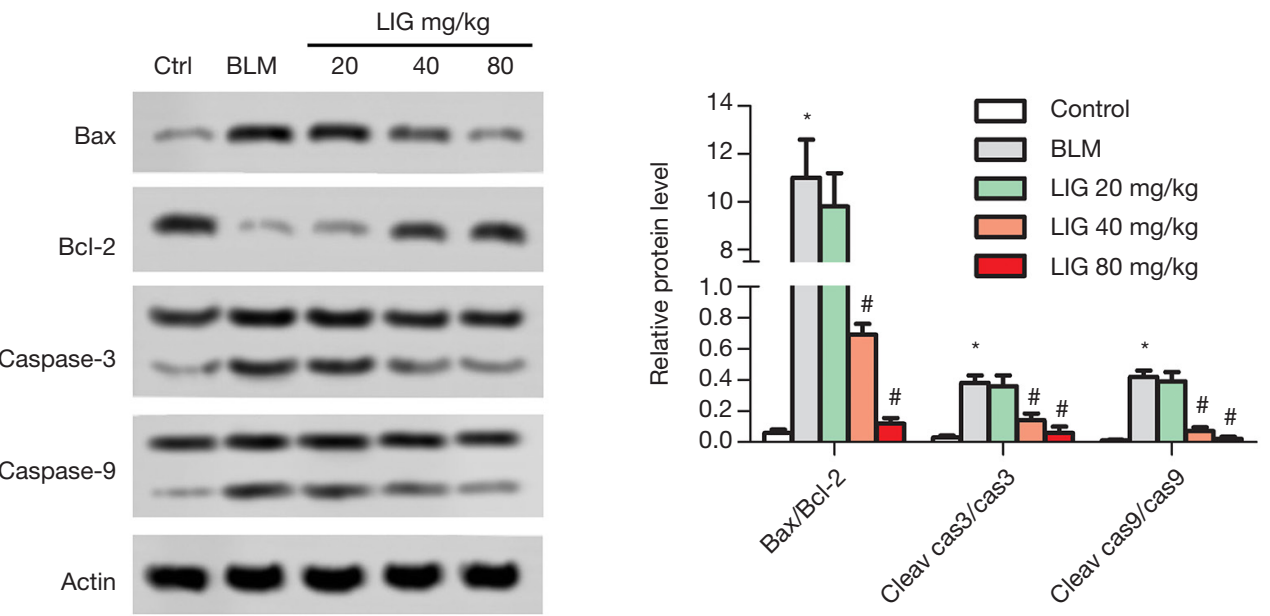

Figure 4 Reduced apoptosis with LIG in bleomycin treated rat lung. Rats were pretreated with bleomycin once and treated with an indicated dosage of LIG for 28 days and (A) TUNEL staining of the lung tissue observed under 200x scope and average apoptotic cells (Yellow-stained) per 100 cells counted. (B) Apoptosis related proteins Bax, Bcl-2, Caspase-3, and Caspase-9, were evaluated with western blotting, and the relative expression of proteins was evaluated with Image $\mathrm{J}$ software. $\mathrm{n}=6$ for each group, ${ }^{*} \mathrm{P}<0.05$ versus control and ${ }^{\#} \mathrm{P}<0.05$ versus bleomycin with $t$-test. Data were shown as mean \pm SEM. LIG, ligustilide.

could mutually interact to promote $\mathrm{PF}$, and co-targeting of these processes, especially targeting of TLR4, could be a potentially effective way of therapy (21). LIG was shown to inhibited oxidative stress in multiple ways, including Nrf-2 and ferroportin $(23,24)$. Current studies highlighted the anti-inflammation effects of LIG, primarily through TLR4 and NF- $\kappa B$ (25). In this study, we showed that the anti-oxidative, anti-apoptotic and anti-inflammation effects of LIG also exist in bleomycin-induced PF. As expected, TLR4, MyD88, and NF-кB P65 were inactivated with LIG dose-dependently.

Inflammatory immune response was activated in $\mathrm{PF}$, in which the Th2-type immune response was overactivated, while lung fibroblasts possibly tend to induce a Th-type immune response (9). Regulation of Th1/Th2 immunity was an essential target of treating PF. For example, Valsartan showed a significant protective effect against bleomycin-induced PF, possible through NF- $\mathrm{KB}$ expression and regulation of Th1/Th2 cytokines (10). During the treatment of LIG, the Th1-type immune response was inhibited, and the Th2-type immune response was activated. These results were possible as a result of decreased fibrosis, as indicated with Masson staining.

The pathogenesis of PF remained mostly unclear, and 


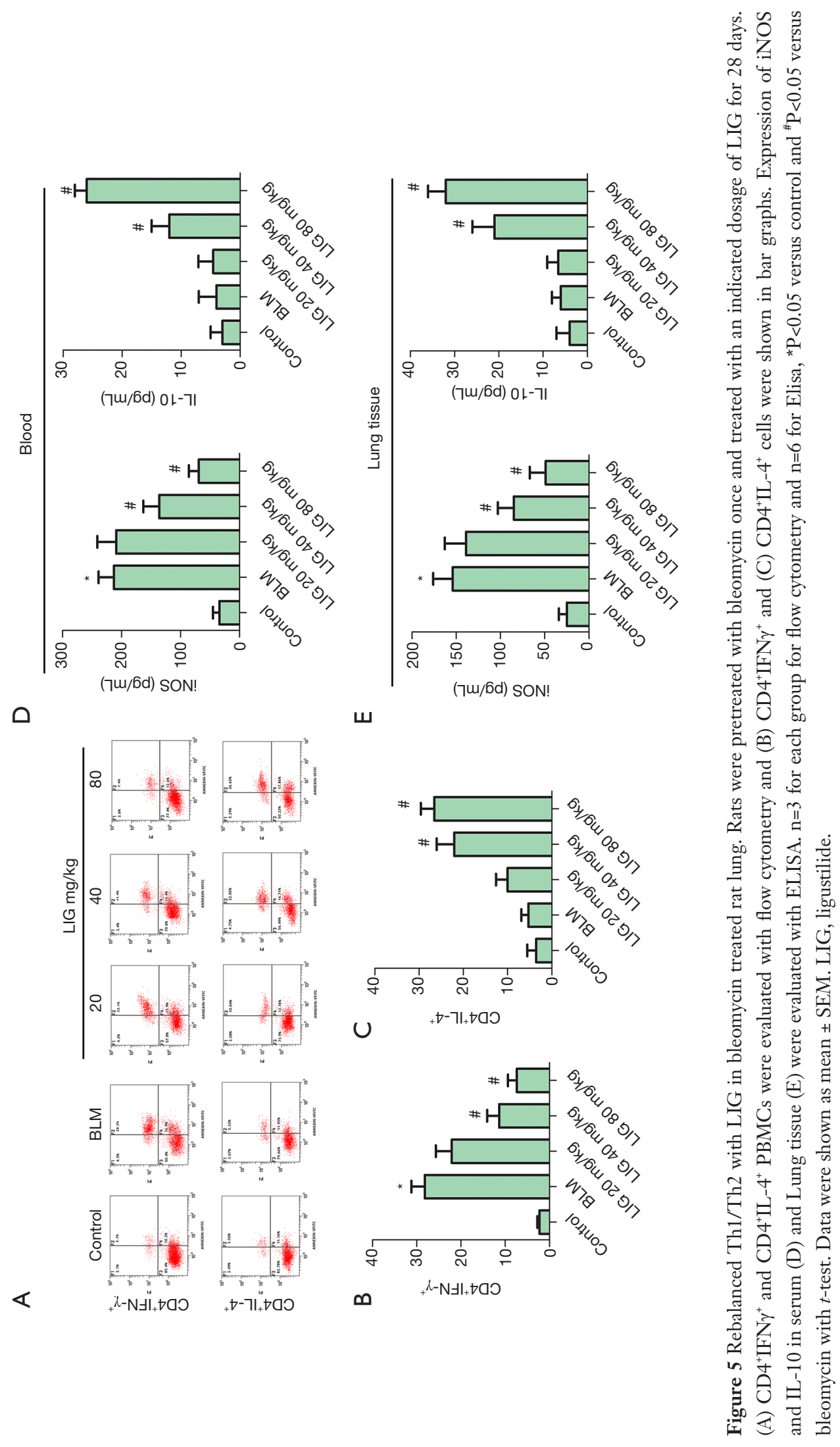


A

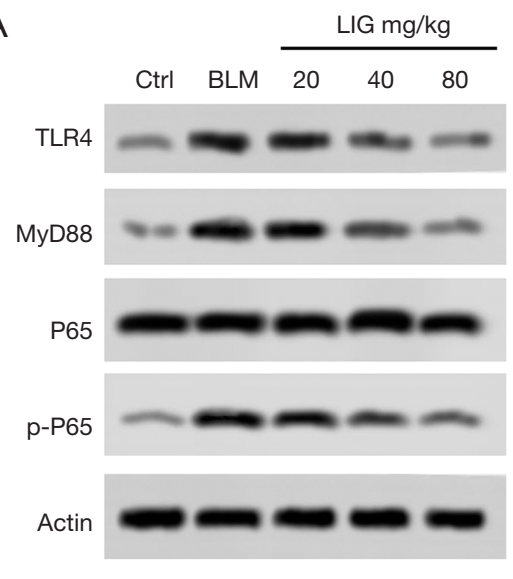

C

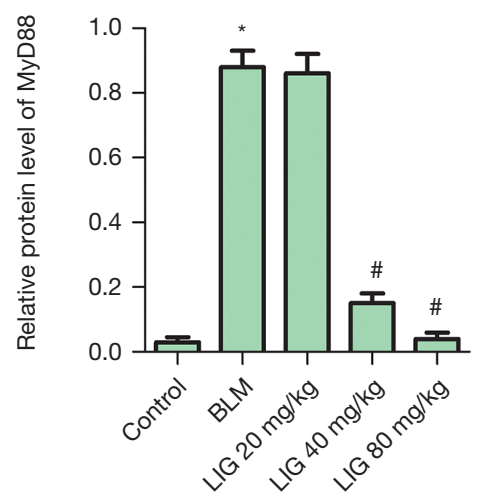

B

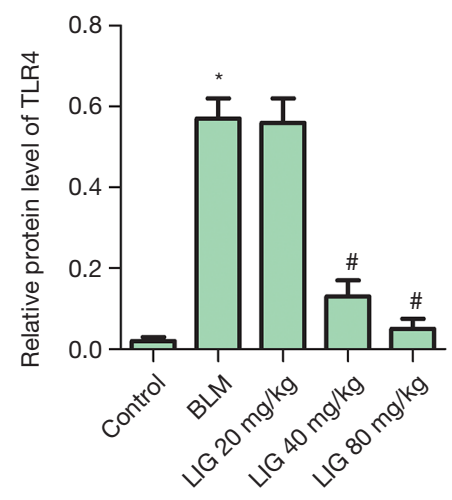

D

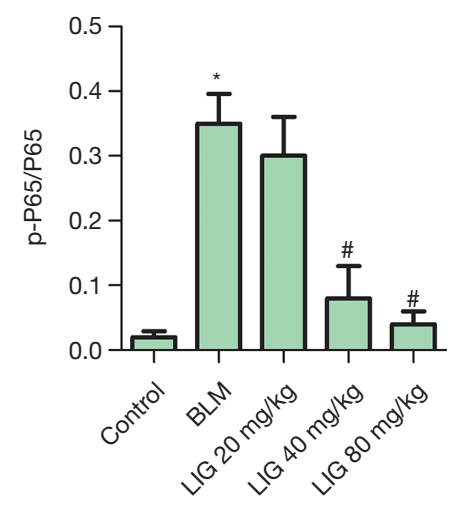

Figure 6 Expression of TLR4, MyD88, and P65 in LIG and bleomycin treated rat lung. Rats were pretreated with bleomycin once and treated with an indicated dosage of LIG for 28 days. (A) Protein expression of TLR-4, MyD88, P65, and p-P65 were tested with western blotting and relative expression levels of LTR4 (B), MyD88 (C), and the ratio of p-P65 to P65 (D) was evaluated with Image J software. n=6 for each group, ${ }^{*} \mathrm{P}<0.05$ versus control and ${ }^{*} \mathrm{P}<0.05$ versus bleomycin with $t$-test. Data were shown as mean $\pm \mathrm{SEM}$. LIG, ligustilide.

the treatment of PF is still limited. Current research and this paper indicated the benefits of TLR4/MyD88/NFкB P65 targeting in PF. However, there may be another mechanism of action for LIG in treating PF, such as referring myofibroblast differentiation, matrix deposition, fibroblast proliferation, senescence-associated secretory phenotype, or regulation of microRNAs (26). So the effects and mechanisms characterized here could merely result in form ameliorated fibrosis but not the reason by which fibrosis ameliorated.

In conclusion, LIG ameliorated bleomycin-induced $\mathrm{PF}$ with improved ventilation, reduced fibroblast, reduced oxidative stress and apoptosis, and rebalanced Th1/Th2 immunity, possibly through TLR4/MyD88/NF-кB P65 signaling.

\section{Acknowledgments}

Funding: None.

\section{Footnote}

Reporting Checklist: The authors have completed the ARRIVE reporting checklist. Available at http://dx.doi. org/10.21037/atm-20-4233

Data Sharing Statement: Available at http://dx.doi. org/10.21037/atm-20-4233

Conflicts of Interest: All authors have completed the ICMJE uniform disclosure form (available at http://dx.doi. 
org/10.21037/atm-20-4233). The authors have no conflicts of interest to declare.

Ethical Statement: The authors are accountable for all aspects of the work in ensuring that questions related to the accuracy or integrity of any part of the work are appropriately investigated and resolved. All animal experiments complied with the guidelines developed by the Health Department of Local Government and Experimentation Ethics Committee of the Universities and approved by the Department of Health of the Hong Kong Government and the Animal Ethics Committee of The Chinese University of Hong Kong (Ref: 13/085/GRF-5).

Open Access Statement: This is an Open Access article distributed in accordance with the Creative Commons Attribution-NonCommercial-NoDerivs 4.0 International License (CC BY-NC-ND 4.0), which permits the noncommercial replication and distribution of the article with the strict proviso that no changes or edits are made and the original work is properly cited (including links to both the formal publication through the relevant DOI and the license). See: https://creativecommons.org/licenses/by-nc-nd/4.0/.

\section{References}

1. Mora AL, Rojas M, Pardo A, et al. Emerging therapies for idiopathic pulmonary fibrosis, a progressive age-related disease. Nat Rev Drug Discov 2017;16:755-72.

2. Nakamura M, Okamoto M, Fujimoto K, et al. A retrospective study of the tolerability of nintedanib for severe idiopathic pulmonary fibrosis in the real world. Ann Transl Med 2019;7:262.

3. Hambly N, Goodwin S, Aziz-Ur-Rehman A, et al. A cross-sectional evaluation of the idiopathic pulmonary fibrosis patient satisfaction and quality of life with a care coordinator. J Thorac Dis 2019;11:5547-56.

4. Fukumoto J, Leung J, Cox R, et al. Oxidative stress induces club cell proliferation and pulmonary fibrosis in Atp8b1 mutant mice. Aging (Albany NY) 2019;11:209-29.

5. O'Dwyer DN, Moore BB. Animal Models of Pulmonary Fibrosis. Methods Mol Biol 2018;1809:363-78.

6. Mora AL, Bueno M, Rojas M. Mitochondria in the spotlight of aging and idiopathic pulmonary fibrosis. J Clin Invest 2017;127:405-14.

7. Yan B, Ma Z, Shi S, et al. Sulforaphane prevents bleomycininduced pulmonary fibrosis in mice by inhibiting oxidative stress via nuclear factor erythroid 2related factor2 activation. Mol Med Rep 2017;15:4005-14.

8. Gille T, Didier M, Rotenberg C, et al. Intermittent Hypoxia Increases the Severity of BleomycinInduced Lung Injury in Mice. Oxid Med Cell Longev 2018;2018:1240192.

9. Sumida A, Hasegawa Y, Okamoto M, et al. TH1/TH2 immune response in lung fibroblasts in interstitial lung disease. Arch Med Res 2008;39:503-10.

10. Mojiri-Forushani H, Hemmati AA, Khodadadi A, et al. Valsartan attenuates bleomycin-induced pulmonary fibrosis by inhibition of NF-kappaB expression and regulation of Th1/Th2 cytokines. Immunopharmacol Immunotoxicol 2018;40:225-31.

11. McKee AS, Mack DG, Crawford F, et al. MyD88 dependence of beryllium-induced dendritic cell trafficking and CD4 $\left(^{+}\right)$T-cell priming. Mucosal Immunol 2015;8:1237-47.

12. Zhou Y, Ming J, Li Y, et al. Ligustilide attenuates nitric oxide-induced apoptosis in rat chondrocytes and cartilage degradation via inhibiting JNK and p38 MAPK pathways. J Cell Mol Med 2019;23:3357-68.

13. Zhao DY, Yu DD, Ren L, et al. Ligustilide protects PC12 cells from oxygen-glucose deprivation/reoxygenationinduced apoptosis via the LKB1-AMPK-mTOR signaling pathway. Neural Regen Res 2020;15:473-81.

14. Ma J, Mei J, Lu J, et al. Ligustilide promotes apoptosis of cancer-associated fibroblasts via the TLR4 pathways. Food Chem Toxicol 2020;135:110991.

15. Zhao LX, Du JR, Zhou HJ, et al. Differences in Proinflammatory Property of Six Subtypes of Peroxiredoxins and Anti-Inflammatory Effect of Ligustilide in Macrophages. PLoS One 2016;11:e0164586.

16. Ma J, Xu Y, Zheng Q, et al. Ligustilide inhibits the activation of cancer-associated fibroblasts. Life Sci 2019;218:58-64.

17. Gao Y, Chen R, Gu N, et al. Effects of ligustilide on the extracellular recombinant human heat shock protein 60 induced inflammatory reactions in the THP-1 cells and the related mechanisms. Zhonghua Xin Xue Guan Bing Za Zhi 2016;44:793-8.

18. Li L, Huang W, Li K, et al. Metformin attenuates gefitinib-induced exacerbation of pulmonary fibrosis by inhibition of TGF-beta signaling pathway. Oncotarget 2015;6:43605-19.

19. Trias EL, Hassantoufighi A, Prince GA, et al. Comparison of airway measurements during influenza-induced tachypnea in infant and adult cotton rats. BMC Pulm Med 2009;9:28. 
20. Kabel AM, Estfanous RS, Alrobaian MM. Targeting oxidative stress, proinflammatory cytokines, apoptosis and toll like receptor 4 by empagliflozin to ameliorate bleomycin-induced lung fibrosis. Respir Physiol Neurobiol 2020;273:103316.

21. Tian R, Zhu Y, Yao J, et al. NLRP3 participates in the regulation of EMT in bleomycin-induced pulmonary fibrosis. Exp Cell Res 2017;357:328-34.

22. Song C, He L, Zhang J, et al. Fluorofenidone attenuates pulmonary inflammation and fibrosis via inhibiting the activation of NALP3 inflammasome and IL-1beta/IL1R1/MyD88/NF-kappaB pathway. J Cell Mol Med 2016;20:2064-77.

Cite this article as: Luo S, Gong J, Cao X, Liu S. Ligustilide modulates oxidative stress, apoptosis, and immunity to avoid pathological damages in bleomycin induced pulmonary fibrosis rats via inactivating TLR4/MyD88/NF-KB P65. Ann Transl Med 2020;8(15):931. doi: 10.21037/atm-20-4233
23. Zhu Y, Zhang Y, Huang X, et al. Z-Ligustilide protects vascular endothelial cells from oxidative stress and rescues high fat diet-induced atherosclerosis by activating multiple NRF2 downstream genes. Atherosclerosis 2019;284:110-20.

24. Zhang YT, Li FM, Guo YZ, et al. (Z)-ligustilide increases ferroportin1 expression and ferritin content in ischemic SH-SY5Y cells. Eur J Pharmacol 2016;792:48-53.

25. Han L, Liu DL, Zeng QK, et al. The neuroprotective effects and probable mechanisms of Ligustilide and its degradative products on intracerebral hemorrhage in mice. Int Immunopharmacol 2018;63:43-57.

26. Lederer DJ, Martinez FJ. Idiopathic Pulmonary Fibrosis. N Engl J Med 2018;378:1811-23. 